\title{
Epidemiology as a Practical Resource to Examine the Hype \& Deliver Reliable Messages
}

\section{Sophia Anyatonwu}

Epidemiology and Preparedness, Texas Department of State Health Services, Austin, Texas, United States

Objective

Epidemiologists will be better prepared to serve as a practical resource within their communities and spheres of influence by taking the time to examine data sources behind and implications of news stories and studies that are being widely circulated.

\section{Introduction}

It can be difficult to distinguish between truth, half-truth, fiction, and misinformation as we watch the news, read headlines, and scroll through various social media feeds. Fortunately, epidemiologists have the tools needed to serve as a practical resource for colleagues, partners, and communities. The Scrutinizer Challenge is an opportunity for epidemiologists to tackle at least one news story or study a month that is relevant to public health. The goal is that we would do the research necessary to examine data sources and implications of news stories and studies. This process can help us deliver consistent and reliable messages to share with colleagues, partners, and communities. It also provides an opportunity for epidemiologists that practice in different settings to consolidate resources and develop working relationships that may be needed to more thoroughly examine issues.

\section{Methods}

The Scrutinizer Challenge project was launched in January 2018 and introduced to Texas Public Health Association (TPHA) Epidemiology Section members. Participants were asked to select a headline or study to scrutinize. They were provided a guidance document with 10-25 questions to help identify and determine the credibility of data sources, compare these sources to claims being made, and assess overall implications of the news story or headline. Lastly, participants were asked to submit an actionable summary or end product that could be shared with colleagues, a local partner, or the general public. Scrutinizer Challenge project submissions were shared in the Epidemiology Section Newsletter or distributed to members as an educational resource.

\section{Results}

Three Scrutinizer Challenges were submitted between January 2018 and July 2018. News stories and study topics that were scrutinized addressed maternal mortality and morbidity in the United States, social media and population-level behavior change, and supplemental vitamins and minerals for disease prevention and treatment. The actionable summaries that were submitted were aimed at healthcare providers, researchers, and the general public. Limited but positive feedback was provided for each submission. Sources were found to be mostly credible for each news story or study, however, 2 out of 3 headlines did not support the claims made in the news story or study.

\section{Conclusions}

The Scrutinizer Challenges that have been submitted so far indicate that headlines can make incomplete or inaccurate claims even when credible sources are provided. This preliminary finding supports the need for epidemiologists to serve as a practical resource in their spheres of influence and communities, so that they can help cut through the hype and share reliable messages.

ISDS Annual Conference Proceedings 2019. This is an Open Access article distributed under the terms of the Creative Commons AttributionNoncommercial 4.0 Unported License (http://creativecommons.org/licenses/by-nc/3.0/), permitting all non-commercial use, distribution, and reproduction in any medium, provided the original work is properly cited. 